\title{
Spatially resolved photocurrent measurements of organic solar cells: tracking water ingress at edges and pinholes
}

\author{
Krishna Feron ${ }^{a, b,{ }^{*}}$, Timothy J. Nagle ${ }^{a}$, Lynn J. Rozanski ${ }^{a}$, Bill B. Gong ${ }^{c}$, Christopher J. Fell ${ }^{a, b}$ \\ a CSIRO Energy Technology, Newcastle, NSW 2300, Australia \\ buniversity of Newcastle Centre for Organic Electronics, Newcastle, NSW 2308, Australia \\ 'Mark Wainwright Analytical Centre, University of New South Wales, Sydney, NSW 2052, \\ Australia \\ *Corresponding author. Tel.: +61249606248; Fax: +61249606021; E-mail address: \\ Krishna.Feron@csiro.au
}

\begin{abstract}
Many degradation mechanisms in organic solar cells are underpinned by the ingress of water at certain points. We demonstrate the use of a photocurrent mapping technique to examine the diffusive ingress of water at the edges of a cell and at pinholes in the cathode layer. A diffusion model applied to the experimental results leads to a value of $(5.0 \pm 2.7) \times 10^{-6} \mathrm{~cm}^{2} \mathrm{~s}^{-1}$ for the diffusivity of water in the poly(3,4-ethylenedioxythiophene):poly(styrene sulfonate) layer. The existence of this layer increases the rate of cell degradation by a factor of ten. The literature is inconclusive with respect to which entry pathway for water is the most important. We find that (a) water ingress clearly occurs at pinholes and at the device edges, rather than uniformly through the cathode film; (b) that ingress at pinholes has the same time dependence as ingress at the edges, however the absolute rate depends on the size of the pinhole; and (c) that degradation at the device edges is faster than even at large pinholes, except where the cathode layer extends beyond the boundary of the active cell. It follows that the lateral architecture of the cell can significantly impact the degradation of efficiency in the device, and we quantify this effect. We demonstrate that the photocurrent mapping method does not require complete local annihilation of device performance in order to track the ingress of water. This means it may be valuable for the study of water ingress through encapsulants in commercial or near-commercial devices.
\end{abstract}

Keywords: organic solar cell; degradation; light beam induced current; diffusion; water; PEDOT:PSS

\section{Introduction}

Solar cells based on organic materials may offer an improvement over traditional photovoltaics, due to their potential for low-cost, renewable feedstock and low manufacturing costs. The performance of organic photovoltaic (OPV) devices continues to improve, with the milestone of $10 \%$ efficiency recently achieved [1]. However, OPV durability remains well short of the requirement for a commercially successful product.

Environmental degradation of OPV devices occurs via a number of mechanisms. These include photooxidation of the organic active materials; electrochemical reactions between 
organic layers and metallic electrodes; electrode corrosion; and physical effects, such as delamination $[2,3]$. These degradation modes can occur concurrently, making it difficult to attribute the observed performance decay to any one cause [4], however most of the degradation mechanisms are underpinned by the ingress of water and oxygen into the device. In this work, we demonstrate the use of a photocurrent mapping technique to examine diffusive ingress of water in OPV devices with the conventional structure: indium-tin-oxide/poly(3,4-ethylenedioxythiophene):poly(styrene sulfonate)/poly(3-hexylthiophene)-[6-6]-phenyl C61-butyric acid methyl ester /cathode (ITO/PEDOT:PSS/(P3HT-PCBM/cathode). If the cathode layer is made of aluminium, the primary degradation mechanism in this device type is well known to be oxidation of the cathode at the internal interface. The PEDOT:PSS layer promotes this reaction, since its hygroscopic nature facilitates rapid uptake of water from the atmosphere $[5,6]$. The formation of an aluminium oxide layer at the cathode interface leads to locally-complete inhibition of charge extraction [7]. In addition, we observe that this oxidation reduces the fill-factor of the cell by decreasing the area available for series conductance.

It has been previously reported $[7,8]$ that the diffusion of water into OPV devices occurs preferentially "through the grain structure" of the aluminium film, rather than through pinholes or at the device edge. A water-driven degradation process might therefore be expected to cause a degradation pattern that is laterally uniform across the device. Our results show that for devices with the normal aluminium cathode thickness of around 100 $\mathrm{nm}$, this is not the case. We report results that instead support the observations of Voroshazi et al. [6], who conclude that water mainly diffuses via pinholes in the cathode and via the edges of the device.

Some authors have concluded that contaminant ingress via the edge of the device is of secondary importance compared to ingress at pinholes [8-11]. Conversely, we find that edges appear to be the principal pathway for ingress. Others have also observed this, especially for small-area devices [6,12-14]. Voroshazi et al. [6] concluded that the impact of the perimeter:area ratio of the device affects the device lifetime. In this work, we demonstrate the use of the light-beam induced current (LBIC) technique to determine the diffusivity of water into OPV cells through pinholes and at the edges of the device. We show that not only the device lifetime but also the dominant diffusion pathway depends on the lateral design of the cell. In particular, the extent to which the photoactive perimeter is defined by the cathode can impact the cell lifetime by more than an order of magnitude and must always be considered when reporting cell durability results.

Since low work function cathode metals such as aluminium are reactive to oxygen and water [7], inverted cell geometries (transparent ITO forms the cathode instead of the anode) [15] frequently lead to improved device lifetimes as they allow for the use of more stable electrode materials, e.g. silver. Although a silver electrode is also subject to oxidation, the oxide is generally not detrimental to device performance. Nevertheless, we demonstrate that LBIC can also be used to measure diffusion in devices that use a silver electrode.

Spatially resolved methods for the study of photovoltaics are not new. Techniques such as conductive atomic force microscopy $[16,17]$ and near-field scanning optical microscopy [18] allow mapping of various performance parameters across a solar cell with very high 
resolution. These methods are valuable for investigating micron-sized areas of the cell surface, but are less effective for tracking effects that occur over greater length scales. Imaging techniques such as lock-in thermography [19], photo- and electroluminescence [14] and light-beam induced current (LBIC) [20] are more suitable for analysing larger areas.

LBIC is a well-known low-resolution technique for observing variations in performance across larger areas of a solar cell. LBIC has been used to identify spatial features in OPV devices that vary depending on the device's preparation process [13]. Krebs et al. [21] used the technique to investigate printed metal electrodes, while Galagan et al. looked at the impact of current collecting grids [22]. The technique has also previously been used to study degradation in organic cells. Kroon et al. [23] observed a decline in photocurrent that decreased homogeneously across the entire cell area. Rösch et al. [14] used LBIC with other imaging techniques to investigate degradation in different device structures. Small OPV modules have also been studied using LBIC $[24,25]$.

Although we have used non-encapsulated devices for this study, the LBIC approach is equally applicable to encapsulated devices, where it could be a valuable tool for studies of encapsulant performance.

\section{Experimental details}

\subsection{Organic photovoltaic device fabrication}

OPV devices with the structure indicated above were fabricated on glass substrates with patterned ITO (15 $\Omega / s q$, Kintec) after cleaning with a series of solvent sonications and a 15 -minute plasma treatment in a mixture of air and pure oxygen. A $35( \pm 5) \mathrm{nm}$ layer of PEDOT:PSS was applied by spincasting, then annealed for 10 minutes at $140{ }^{\circ} \mathrm{C}$. The annealing was conducted in an inert environment, as were all subsequent fabrication steps. A 1:1 blend of P3HT and PCBM was dissolved in chlorobenzene at $15 \mathrm{mg} / \mathrm{mL}$. The solution was filtered $(0.2 \mu \mathrm{m})$, and then spincast on top of the annealed PEDOT:PSS with thickness of $80 \mathrm{~nm}$ to form the active layer of the device. A $100 \mathrm{~nm}$ thick cathode layer (either silver or aluminium) was deposited by evaporation through a mask, such that each sample contained four independent test cells with a common ITO contact. The samples were then annealed for 4 minutes at $140^{\circ} \mathrm{C}$. In this configuration, shown in Fig. 1, the $2 \mathrm{~mm} \times 10 \mathrm{~mm}$ active area of each test cell is defined at the long edges by the dimensions of the metal cathode layer, and at the short edges by the width of the ITO bottom contact. This configuration will be shown to have implications for the durability of the test cells. 


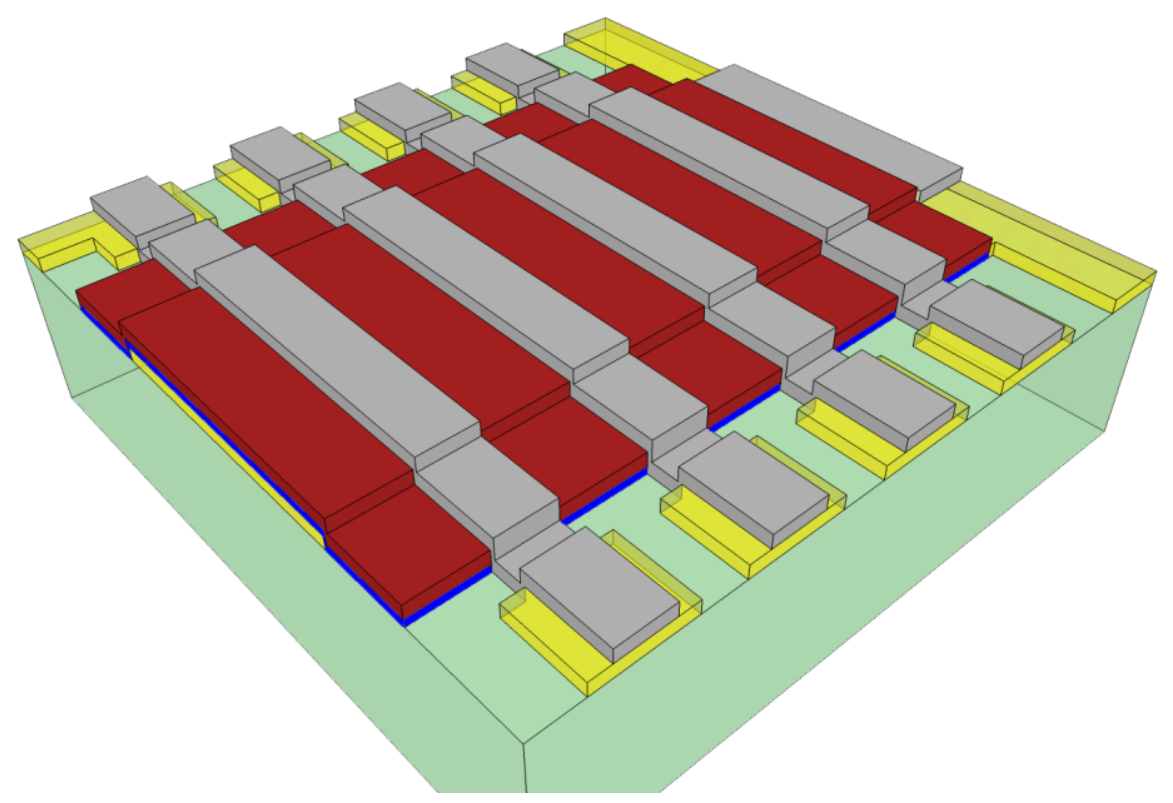

Figure 1: Layout for OPV devices used in this study. Yellow indicates ITO, red the active layer, blue the PEDOT:PSS layer and grey the metal cathode.

To evaluate the relative increase in water uptake due to the PEDOT:PSS layer, several devices were made without this layer. A number of devices were also encapsulated using a glass cover slide and a UV curable epoxy (Lens Bond, Emsdiasum) so that cell performance could be characterised without the effects of degradation. For the encapsulated devices, mean values of the solar cell parameters open-circuit voltage $\left(V_{\text {oc }}\right)$, short-circuit current $\left(J_{\text {sc }}\right)$, fill-factor ( $F F$ ) and efficiency ( $\eta$ ), obtained in CSIRO's PV measurement laboratory (Class AAA solar simulator, $1000 \mathrm{Wm}^{-2}$, no temperature control in this case), were as follows:

- devices with PEDOT:PSS and an aluminium cathode; $V_{\mathrm{oc}}=650 \mathrm{mV}, \mathrm{J}_{\mathrm{sc}}=9.6 \mathrm{mAcm}^{-2}$, $\mathrm{FF}=0.48, \eta=3 \%$

- devices with no PEDOT:PSS layer and with an aluminium cathode; $V_{\text {oc }}=410 \mathrm{mV}, J_{\mathrm{sc}}=$ $6.1 \mathrm{mAcm}^{-2}, \mathrm{FF}=0.34, \eta=0.9 \%$

- devices with PEDOT:PSS and a silver cathode; $V_{\mathrm{oc}}=420 \mathrm{mV}, \mathrm{J}_{\mathrm{sc}}=8.6 \mathrm{mAcm}^{-2}, \mathrm{FF}=$ $0.40, \eta=1.4 \%$.

The remainder of the devices were fabricated without encapsulation. The non-encapsulated devices were kept in the dark under an inert atmosphere until used for either photocurrent mapping experiments (LBIC) or current-voltage (I-V) measurements. The transfer of devices to the LBIC apparatus exposed those samples to air for 1-10 minutes.

\subsection{Light beam-induced current system}

A schematic diagram of the LBIC apparatus is shown in Fig. 2. A laser diode (635 nm, Thorlabs) is electronically modulated at $191.1 \mathrm{~Hz}$ and the output is pigtailed into a single-mode optical fibre. The other end of the fibre is coupled into an assembly containing a collimating lens and a focusing lens. The assembly is attached to a precision translation stage and may be moved along the axis of the beam to change the position of the focal plane. Our apparatus is capable of producing a spot radius down to $3.3 \mu \mathrm{m}$. The solar cell is 
mounted on a set of two orthogonal, computer-controlled translation stages (Thorlabs) and contacted with four probes to allow for separate voltage- and current-sensing circuits. The modulated photocurrent induced by the laser beam in the device under test is amplified by a current-to-voltage preamplifier (Stanford Research, SR570) and detected with a lock-in amplifier (Stanford Research, SR830). The use of a silicon reference solar cell (Fraunhofer ISE) for calibration allows the measurements to be presented as a map of apparent external quantum efficiency (EQE). This calibration assumes a linear photocurrent response in the test device, which is approximately true for spot radii larger than $75 \mu \mathrm{m}$. The use of spot sizes smaller than this results in a very high local irradiance (up to around 1000 suns). Under such conditions, our device response becomes nonlinear. EQE values taken from low-resolution scans are therefore approximately correct, but values taken from high-resolution scans, while approximately in proportion within one scan, may not be correct in an absolute sense.

For several of the tests described here, the apparatus was placed inside a glovebag (Sigma Aldrich, Atmosbag Z530220) to allow for measurements in a controlled atmosphere. Measurements were conducted at different times under different ambient atmospheres, including laboratory air, dry air, dry nitrogen and wet nitrogen. The latter was achieved by bubbling nitrogen through a cylindrical vessel of water, which produced a relative humidity that could be varied by changing the volume of water in the cylinder. The relative humidity in the glovebag was monitored using a hygrometer (Lambrecht).

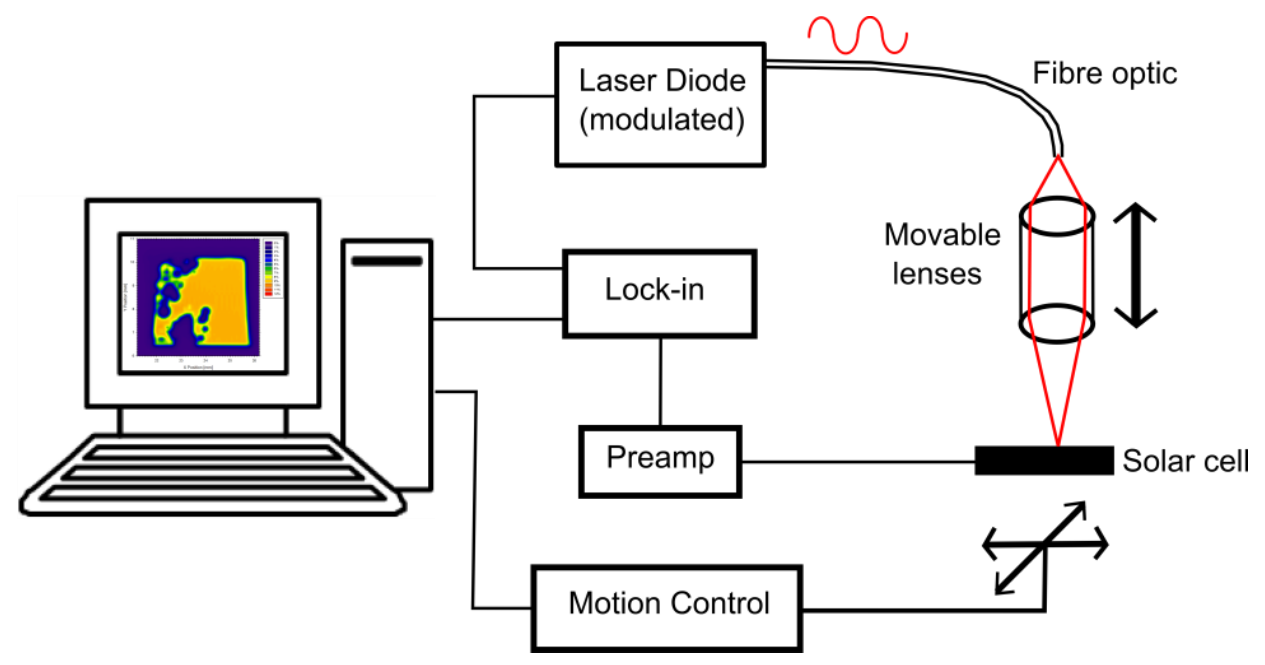

Figure 2: Schematic diagram of the LBIC apparatus

\section{Results and discussion}

\subsection{General observations of encapsulated devices}

Fig. 3 presents quantum efficiency maps for an encapsulated device. Over most of the device area, the EQE is approximately $10 \%$, which is in agreement with whole-cell measurements made at the same wavelength in a separate measurement system. This level of response reflects the relatively weak light absorption in P3HT-PCBM OPV devices at $635 \mathrm{~nm}$ and is adequate for photocurrent mapping measurements. Fig. 3a shows the 
photocurrent response over the entire $2 \mathrm{~mm} \times 10 \mathrm{~mm}$ cell area, measured with a spot radius of $100 \mu \mathrm{m}$. Figs. 3b and 3c show subregions of this cell, measured with a spot radius of $10 \mu \mathrm{m}$. All three scans indicate areas in which little or no photocurrent is generated. These areas are perfectly circular and vary in diameter from 25-250 $\mu \mathrm{m}$. We observe such 'dead zones' in virtually all OPV devices we make. In non-encapsulated devices, we see them grow over time, whereas in encapsulated devices, their size does not change over a period of days. Visual inspections reveal no features that correspond to even the largest dead zone. Hence, we attribute the dead zones to the ingress of a contaminant through small pinhole defects in the aluminium electrode. The high degree of circularity of the dead zones suggests the corresponding holes in the electrode are much smaller. Otherwise, the dead zones would, on at least some occasions, retain non-circular elements of the shape of the hole in the electrode. On this basis, we estimate that the holes in this particular device must have a diameter no larger than a few micrometres, consistent with SEM imaging of pinholes in an aluminium cathode conducted by Krebs et al. [10]. The existence and behaviour of the dead zones in encapsulated devices leads us to believe that the contaminant, which we later confirm to be water, is not fully excluded during our fabrication process.

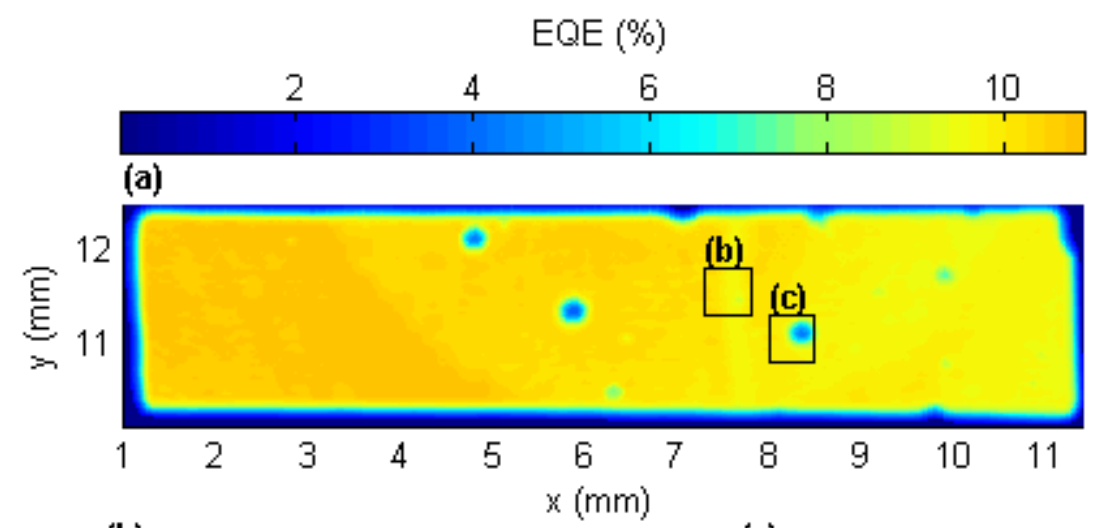

(b)
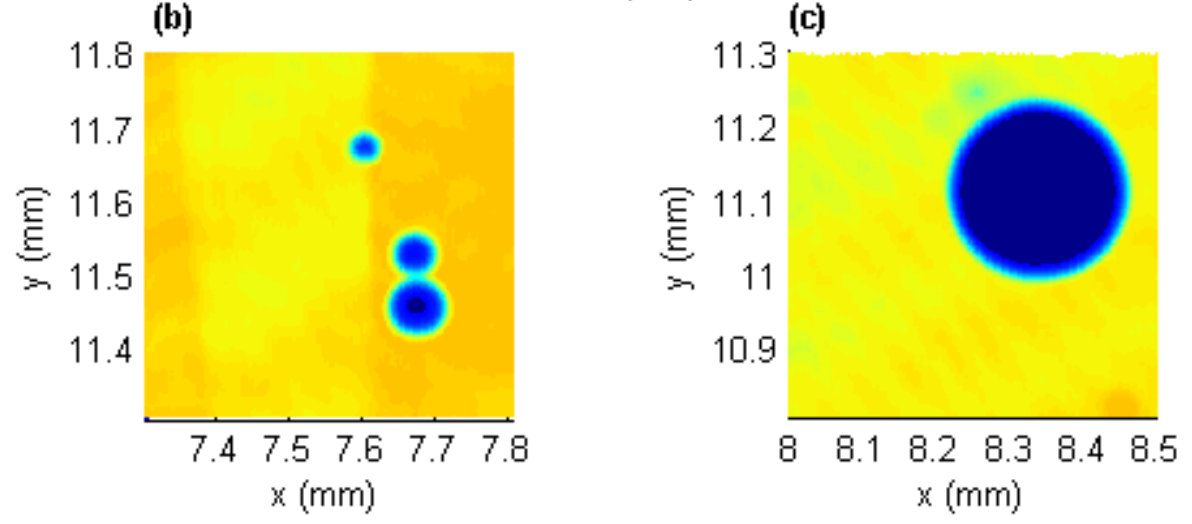

Figure 3: EQE maps of a $2 \mathrm{~mm} \times 10 \mathrm{~mm}$ encapsulated device measured with (a) $100 \mu \mathrm{m}$ spot radius, and $(b-c) 10 \mu \mathrm{m}$ spot radius. Perfectly circular regions of degradation are common and are attributed to pinholes in the metal cathode layer allowing ingress of water

\subsection{General observations in non-encapsulated devices}

Fig. 4 presents four EQE maps for a single device without encapsulation, collected sequentially. During each measurement, the beam moves in a raster pattern: it oscillates from top to bottom in the image, taking 60 minutes to traverse the active length of the cell 
from left to right. Each scan is labelled with a time axis, which represents the time since the device was first exposed to air. Immediately evident in these scans is a progressive decrease in the width of the functional cell. The width of the cell in the image decreases due to complete degradation of the active area at the long edges. This gives rise to a trapezoidal shape as the device degrades symmetrically at the long edges (top and bottom in the images) while the measurement proceeds from left to right. The four scans took 5 hours to complete, after which the active width of the cell was reduced to zero. No other illumination was used during the measurement, demonstrating that this degradation does not require any optical excitation, which is consistent with the observations of Kawano et al. [5].
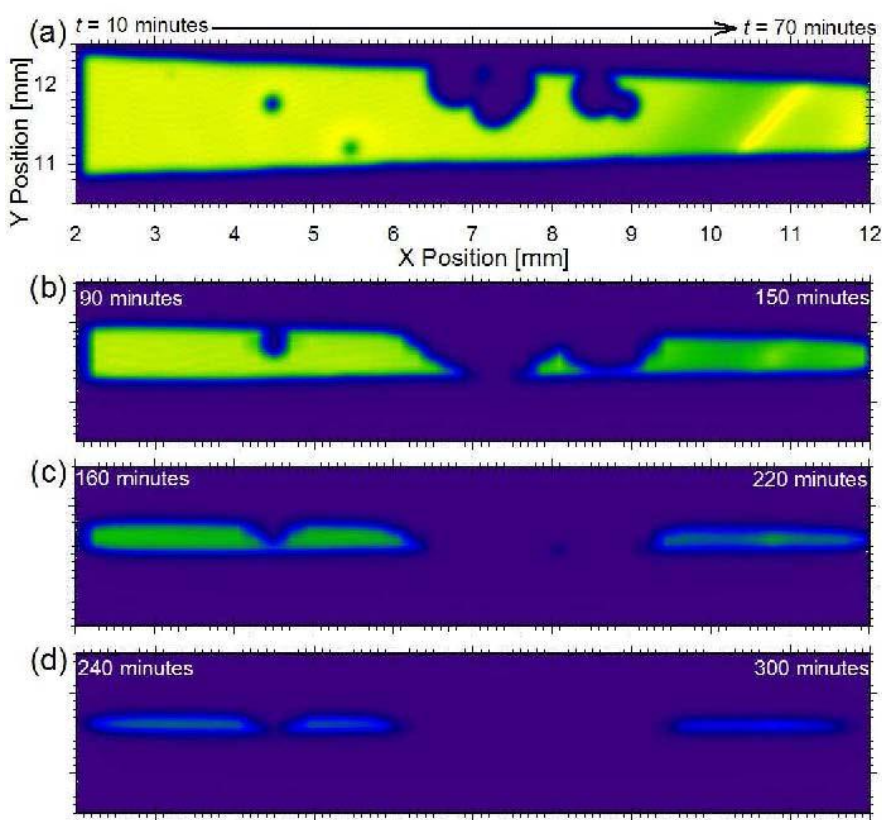

Figure 4: A sequence of four photocurrent maps for a non-encapsulated device exposed to air in the laboratory. The trapezoidal shape is due to degradation progressing as the measurement is being made

The long dimension of the active cell remains constant throughout most of the experiment, indicating that degradation does not occur at the short edges (left and right in the image). The short edges of the device are defined by the absence of ITO, rather than by the edge of the cathode layer. In this case, the cathode layer extends laterally beyond the active edge of the device and would be expected to protect the cell from contaminant ingress at those edges. It follows that the protective nature of the aluminium cathode is of importance to the design of a stable device.

Superimposed on the trapezoidal shape are a number of the circular features, or dead zones, discussed above. These pinhole features can clearly be observed in Fig. 4 to grow over time consistent with previous reports $[7,8,10,11]$. In contrast to these reports and in agreement with others [6,12-14], we observe that the impact of degradation at pinholes is less important, compared with degradation at the edges of the device. This is consistent with the observations of Voroshazi et al. [6], in which the contaminant is identified as water. We have also experimentally confirmed water as the ingressive contaminant in our cells, 
rather than oxygen, and we have included a description of those experiments in the supplementary material.

\subsection{Quantifying the rate of diffusion from edges}

Photocurrent maps such as the ones shown in Fig. 4 allow an analysis of the rate of contaminant ingress into the device. In the following, we make the reasonable assumption that the cathode oxidation reaction occurs very rapidly; and hence, the rate of degradation is limited only by the rate of water diffusion into the device. We also make the further assumption - perhaps more tenuous - that the ingress can be described by Fick's Law. This means that the rate of diffusion at a given temperature is a function only of the difference in contaminant concentration between any two points. We define the degradation length, $x$, as the distance by which degradation has proceeded from the device edge; this is obtained by subtracting the width of the observed photoactive area from the width of the aluminium electrode $(2 \mathrm{~mm})$ and dividing the result by two. A graphical representation of this analysis is included in the supplementary material. Fig. 5 shows the degradation length as a function of time, extracted from the series of photocurrent maps shown in Fig. 4. Points for which the cell width is affected by degradation through pinholes have been discarded for clarity. If we assume the region outside the cell boundary contains the contaminant at a constant concentration, we would expect the degradation length, $x$, to increase with time, $t$, as [26]:

$$
x(t)=\operatorname{erf}^{-1}\left(1-\frac{C^{*}}{C_{0}}\right) \sqrt{4 D t}=a \sqrt{t},
$$

where $D$ is the diffusion coefficient for the contaminant in the device, $C_{o}$ is the contaminant concentration outside the device, $C^{*}$ is the contaminant concentration required to cause complete local degradation, and $a$ is a prefactor that captures all constants. For a device with a PEDOT:PSS hole-transport layer (HTL) we observe a good fit to this relation (Fig. 5), consistent with a degradation mechanism that is caused by diffusion from the edges of the device. Based on this fit we extract a value for $a$ of $7.6 \times 10^{-3} \mathrm{~ms}^{-1 / 2}$ in this device. 


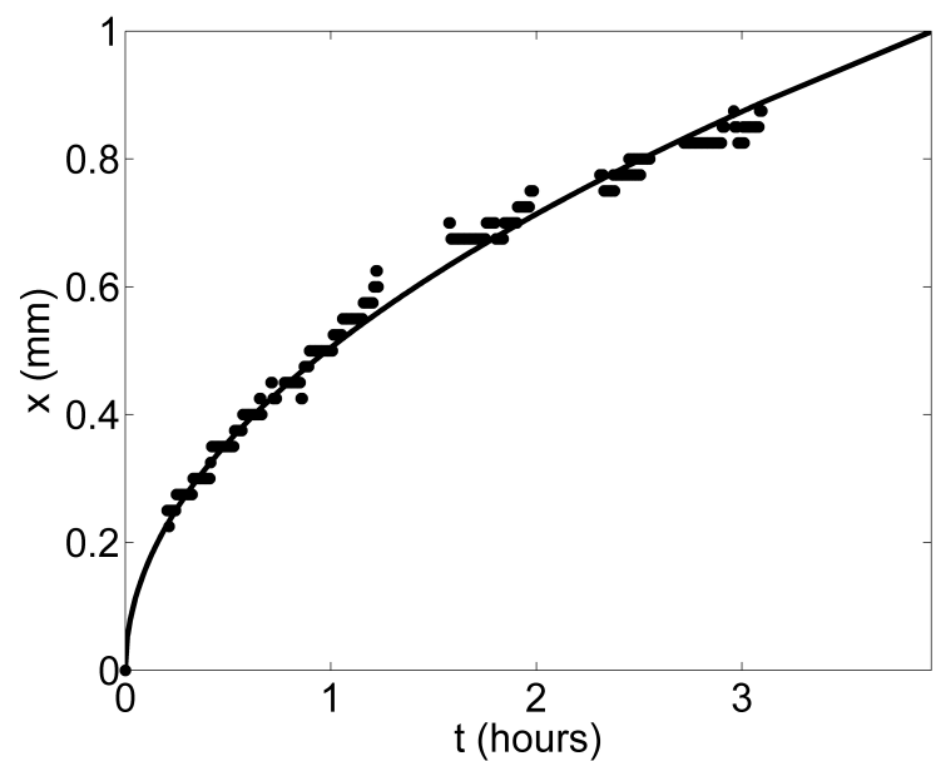

Figure 5: Degradation length, $x$, extracted from photocurrent maps, evolves in a $\sqrt{t}$ relationship with time

The hygroscopic nature of PEDOT:PSS is known to accelerate water uptake and consequently shorten device lifetimes [6]. This has prompted the use of different materials for the HTL that do not influence water uptake [2]. For example, devices that use $\mathrm{MoO}_{3}$ instead of PEDOT:PSS have been shown to exhibit longer lifetimes [6]. In order to determine a lower limit for water uptake we prepared and tested a device without any HTL. The result is shown in Fig. 6b, where the square of the degradation length exhibits a linear evolution with time and fits with zero intercept as expected. The equivalent result for the device with a PEDOT:PSS layer is included at Fig. 6a for comparison.

From the slope of Fig. $6 \mathrm{~b}$ we determine a value of $a$ for the device with no HTL of $6.3 \times 10^{-4}$ $\mathrm{ms}^{-1 / 2}$. Since this parameter effectively describes the rate of diffusion of the contaminant into the device, the result indicates that the presence of a PEDOT:PSS layer has increased the rate of ingress by approximately one order or magnitude.

(a)

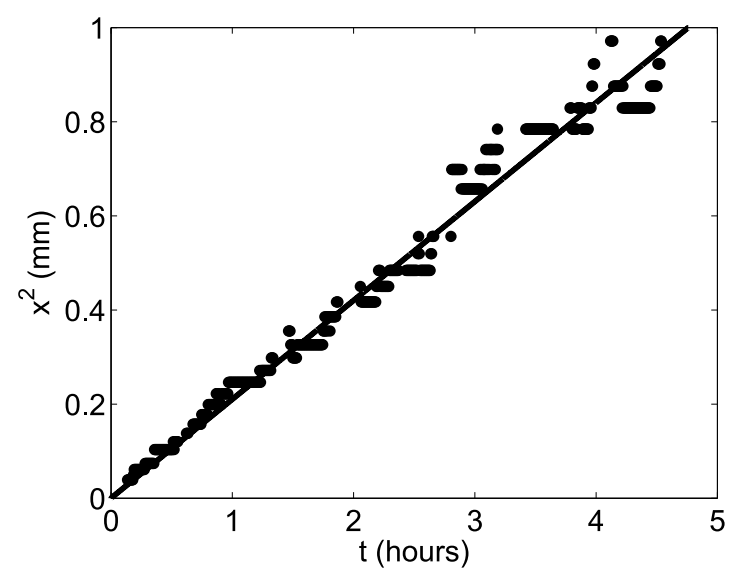

(b)

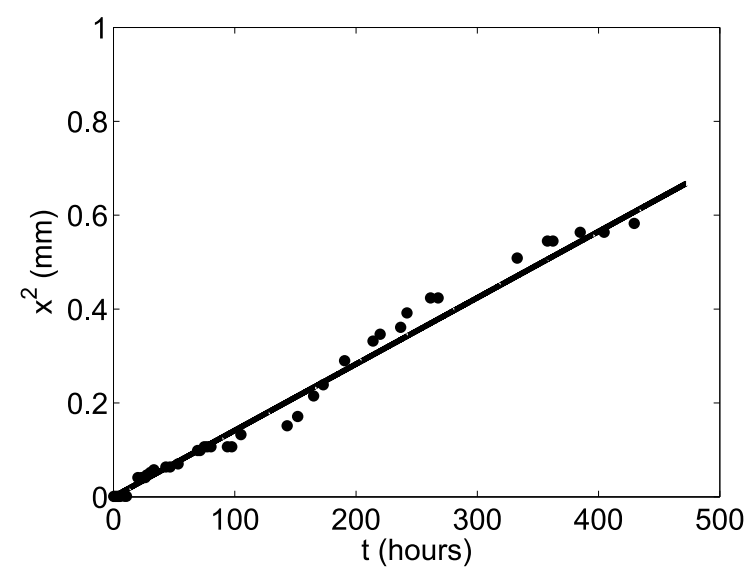


Figure 6: Evolution of the square of degradation length with time for a device using a PEDOT:PSS layer (a) and without any hole-transport layer (b)

\subsection{Extracting the diffusion coefficient}

Equation 1 suggests a known relationship between the growth of degradation and the external contaminant concentration, $C_{0}$. Since we know our contaminant is water, this provides an opportunity to extract further information about the degradation process by repeating the $\mathrm{LBIC}$ measurements under atmospheres with different, known relative humidity levels. Provided temperature and pressure are held constant, the concentration of water in the environment is linearly related to the relative humidity. Hence, for relative humidities $r_{1}$ and $r_{2}$, we expect a variation in the rate prefactor, $a$, according to:

$$
\frac{a_{2}}{a_{1}}=\frac{\operatorname{erf}^{-1}\left(1-f_{2}\right)}{e r f^{-1}\left(1-f_{1}\right)}=\frac{e r f^{-1}\left(1-\frac{r_{1}}{r_{2}} f_{1}\right)}{e r f^{-1}\left(1-f_{1}\right)},
$$

where $f_{\mathrm{n}}=C^{*} / C_{0 \mathrm{n}}$ at the $\mathrm{nth}$ relative humidity.

We repeated the measurement presented in Fig. 5 under controlled conditions, keeping the relative humidity at $54 \%$ for the first 2.2 hours and then at $66 \%$ for the remainder of the experiment. As shown in Fig. 7, the growth of degradation was highly sensitive to the change in humidity. Based on the slopes of the fits in the two humidity regimes, we observe a relative increase in the rate prefactor of $a_{2} / a_{1}=2.58$. On the basis that $C^{*}$ is independent of the external humidity, we can use $a_{2} / a_{1}$ along with Equation 1 to determine a value for the diffusion coefficient for water in PEDOT:PSS. We calculate a value of $D=(5.0 \pm 2.7) \times 10^{-6}$ $\mathrm{cm}^{2} \mathrm{~s}^{-1}$. This result is of the same order as published values for the diffusivity of water in other polymers [27-29].

The results presented in Fig. 7 also allow a value for $C^{*}$ to be extracted using Equation 1. Using the $20^{\circ} \mathrm{C}$ value for the saturation vapour pressure of water, $2.34 \mathrm{kPa}$ [30], we obtain a water concentration $C^{*}=8.4 \pm 0.6 \mathrm{gm}^{-3}$, or around 6 parts per million by weight in the PEDOT:PSS layer. The premise of a value for $C^{*}$ however, implies that below this level no degradation occurs. This seems a somewhat non-physical scenario, particularly given that the equivalent concentration in air represents a relative humidity of $49 \%$. A quick test with a new sample stored under $33 \%$ relative humidity showed that degradation still occurred, albeit more slowly. This supports the assumption that any water in the device leads to degradation at some rate, and hence that the real value for $C^{*}$ is very small as indicated by Voroshazi et al. [6]. It follows that the value we have calculated for $C^{*}$ is too high. This can be explained if we challenge our earlier assumption that the degradation rate depends only on the rate of water diffusion in the PEDOT:PSS layer. In this we have ignored the time taken for any other part of the degradation process, including the passage of water across the P3HT:PCBM layer of the cell. If these other steps require significant time, the outcome is that our estimate of the diffusion constant in PEDOT:PSS is slightly low, and our estimate of $C^{*}$ is high. 


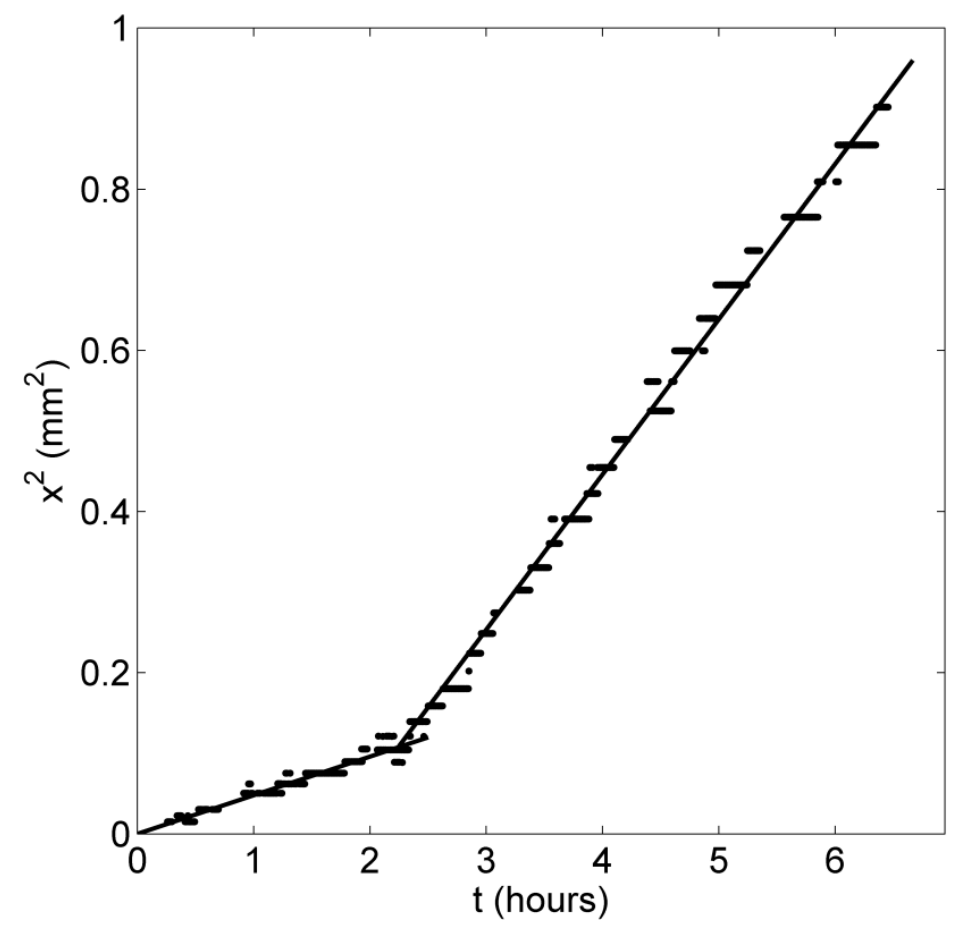

Figure 7: The square of the degradation length as a function of time. At $t=2.2$ hours, the relative humidity was increased from $54 \%$ to $66 \%$ while keeping the temperature constant. The lines indicate fits of Equation 1 to each humidity regime

\subsection{Quantifying ingress at pinholes}

Since some authors [8-11] have observed that degradation due to water ingress at pinholes is more significant than at the device edges, we have examined the LBIC photocurrent maps to measure the rate of device degradation at pinholes. Two large round defects were identified and the growth of their diameter was determined based on twelve consecutive photocurrent maps. The pathway for ingress through a pinhole differs only from that at the device edge by the fact that the pinhole is laterally small. Hence, we assume a $\sqrt{t}$ dependence of the degradation length and a suitable fit takes the form

$$
x=a \sqrt{t}+b
$$

where $b$ is the size of the dead zone at $t=0$. Since this value is likely to correspond to the size of the physical pinhole, a fit of this form means that the pinhole size can be determined even though pinholes are typically smaller than the resolution of the LBIC measurement.

The results are presented in Fig. 8 and these show that the degradation length at pinholes is indeed also proportional to $\sqrt{t}$. Based on the evolution of the degradation length, we determine the diameters of the smaller and larger pinholes to be $66 \mu \mathrm{m}$ and $78 \mu \mathrm{m}$ respectively. The corresponding values for the rate prefactor, $a$, for these are $6.8 \times 10^{-3} \mathrm{~ms}^{-1 / 2}$ and $9.0 \times 10^{-3} \mathrm{~ms}^{-1 / 2}$, respectively. Since this parameter depends on the environmental humidity, a measurement of edge degradation was extracted from the same photocurrent maps for direct comparison. This result is also shown in Fig. 8 and for diffusion at an edge the value for $a$ is determined to be $2.7 \times 10^{-2} \mathrm{~ms}^{-1 / 2}$. 
These results indicate that degradation at the chosen pinholes occurs more slowly than at the device edge, and occurs faster for the larger hole. This is not unexpected, since it is reasonable to assume that diffusive conductance may be limited by the aperture of a pinhole, with diffusion at an edge being equivalent to a pinhole of infinite diameter. Based on the range of sizes for the round features in Figs. 3 and 4, and our further observation, it is clear that pinholes typically occur in a wide range of sizes. Without examining more pinholes we cannot establish a detailed relationship between pinhole size and the rate prefactor. However, since we have selected two of the largest round features we have seen in our devices, we can conclude that degradation at pinholes is unlikely to ever exceed the degradation we observe at the device edge.

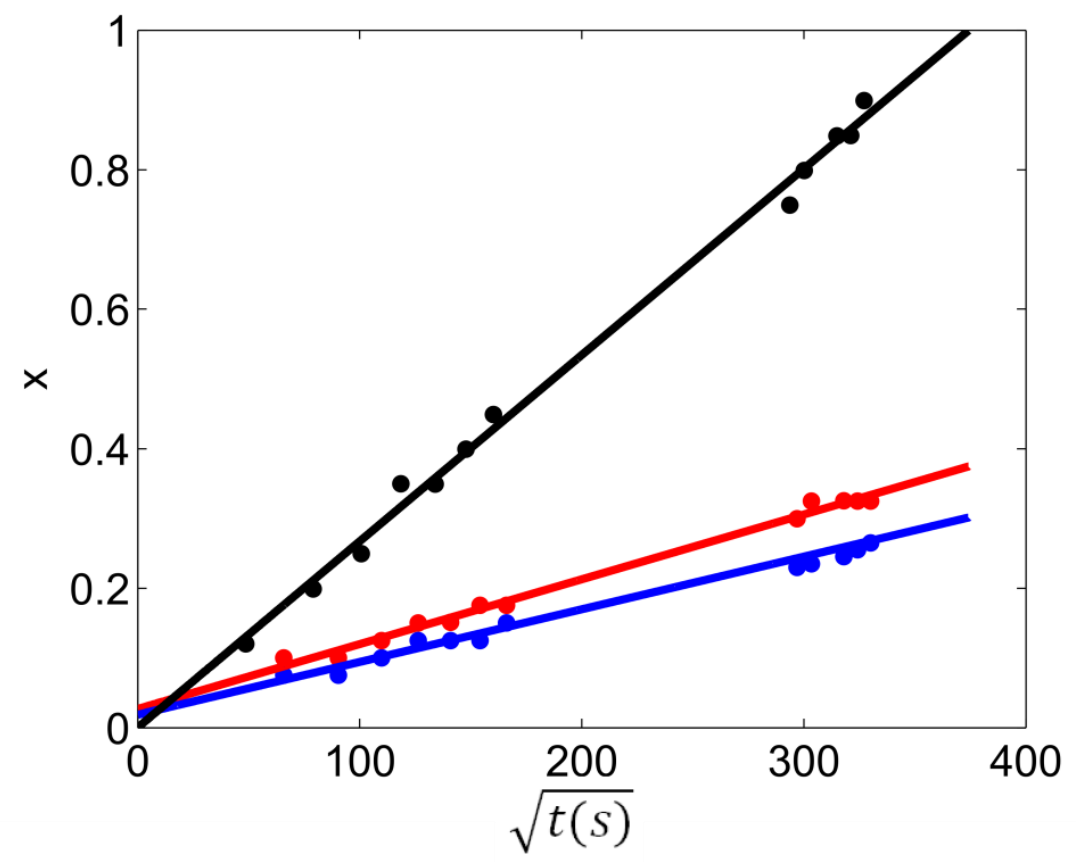

Figure 8: Degradation length against $\sqrt{t}$ for contaminant ingress at a pinhole with $66 \mu \mathrm{m}$ diameter (blue), $78 \mu \mathrm{m}$ diameter (red) and at the device edge for comparison (black)

\subsection{Ingress in devices with a silver cathode}

We have demonstrated that LBIC photocurrent maps can be used to track the diffusion of water in OPV devices with aluminium cathodes, which suffer a total loss of photoactivity in the affected areas. While aluminium is a common cathode in conventional device designs, inverted device designs typically use a silver cathode. Although silver also oxidises, oxides of silver are electrically conductive and have even been used as interfacial layers to improve performance in OPV devices [31,32]. Despite the absence of severe degradation, we have found that LBIC measurements can still be used to track the ingress of water in devices with a silver cathode. A representative line scan for a conventional device with a silver cathode is shown in Fig. 9a. This and other scans indicate a small increase in photocurrent (approx. $3 \%)$, which is symmetrically localised to a particular distance from each long edge of the device. This effect can be seen in Fig. 9a in the form of two small bumps at $y=30$ and $y=31$ 
$\mathrm{mm}$. Analysis of the complete LBIC image (not shown) indicates that these features move inward toward the centre of the cell at approximately the same rate as degradation proceeds in our aluminium cathode devices. Furthermore, the progress of these features exhibits the same $\sqrt{t}$ dependence that we observe in the aluminium cathode devices (see Fig. 9b). We propose that oxidation of the silver cathode is also a result of the ingress of water into the PEDOT:PSS layer, and that the localised increase in photocurrent is due to a favourable temporary oxidation state. The work function of sub-stoichiometric oxides of silver varies with oxygen concentration [33] and our results suggest that the most favourable form of silver oxide is not the final oxidation state, but rather some partial oxidation state that occurs during the oxidation process, proceeding through the cell as water diffuses.
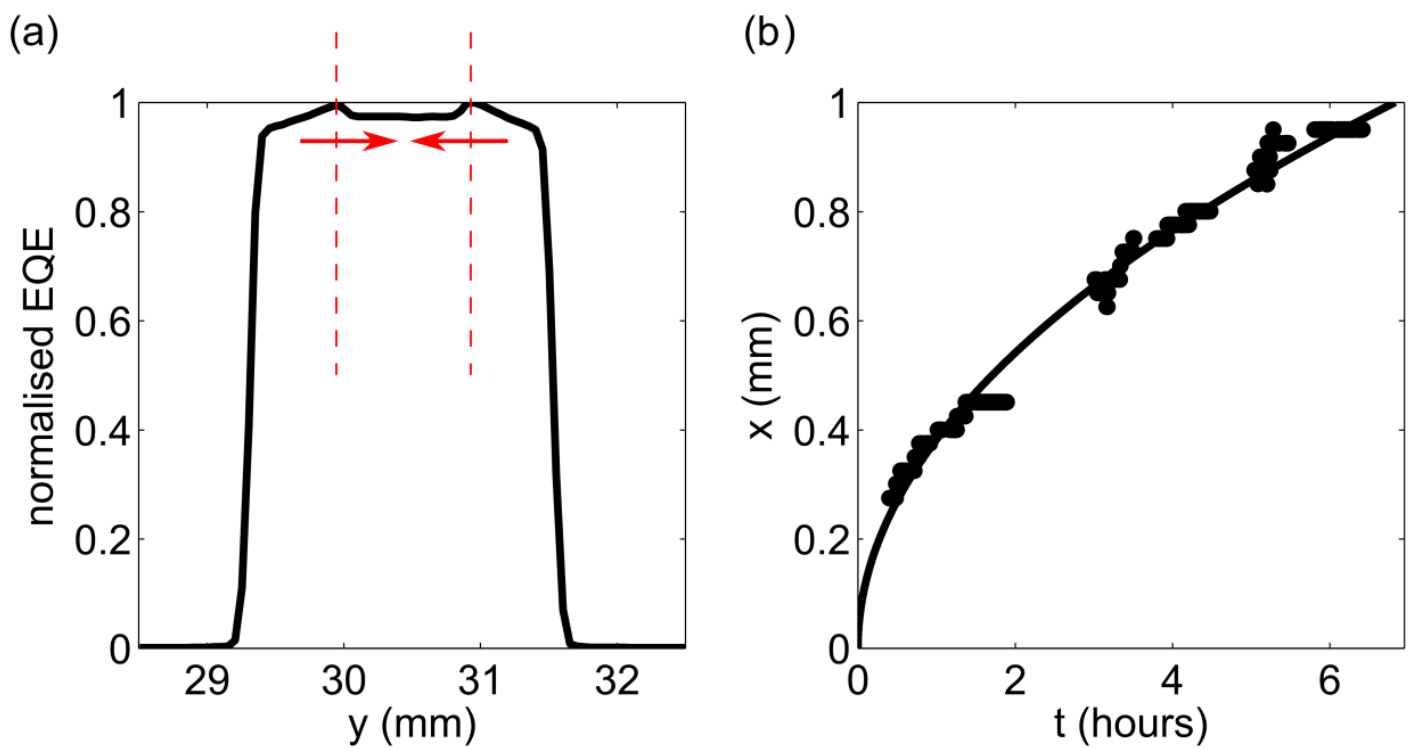

Figure 9: (a) LBIC line scan across a cell with a silver cathode, indicating two symmetrically located regions of slightly increased photocurrent. Area scans (not shown) indicate these features move inward with a $\sqrt{t}$ time dependence as shown in (b)

\subsection{Optimising lateral design for device durability}

In order to relate the growth of local degradation to the decay in power conversion efficiency, fresh devices were prepared without encapsulation for I-V studies. The devices were stored in air and I-V curves were recorded regularly over 24 hours, with the standard photovoltaic parameters extracted from the data. The two parameters most notably affected were the short-circuit current, $I_{\mathrm{sc}}$, and the cell series conductance, $1 / R_{\mathrm{se}}[5,6]$. Fig. 10 shows typical examples of the variation of these two parameters over time. In Fig. 10a, $I_{s c}$ for a cell is seen to decrease linearly with $\sqrt{t}$. For a rectangular cell with an active length that remains constant but an active width decreasing with $\sqrt{t}$, this is precisely what we would expect, based purely on the loss of active cell area. This is a direct result of diffusion from parallel devices edges dominating the overall loss of photocurrent. The variation in cell conductance (Fig. 10b) is equally interesting. Its time dependence also shows a clearly linear decrease with $\sqrt{t}$, with a rate constant identical to that of the photocurrent decay. From this, we infer that the loss of conductive area occurs at the same 
rate as the loss of photoactive area. We conclude that the water-driven oxidation at the cathode interface simultaneously affects both photoactivity and cell conductance.

(a)

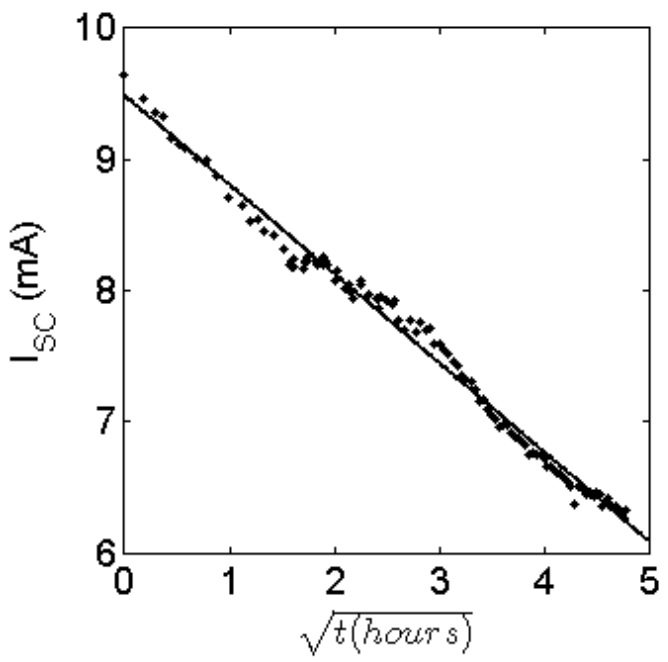

(b)

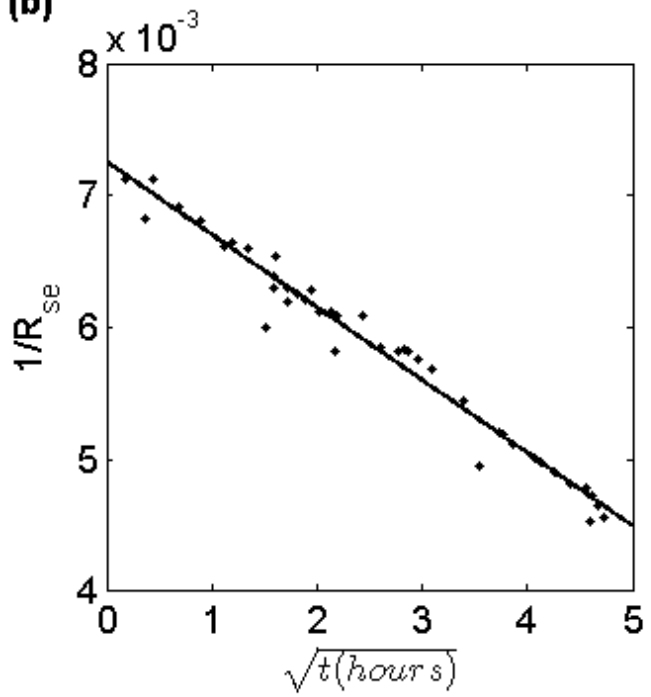

Figure 10: I-V measurements for non-encapsulated OPV cells stored in air indicate that short-circuit current (a) and series conductance (b), decay linearly with $\sqrt{t}$ at the same rate

It is important to remember that this ingress is a direct consequence of the lateral device design we have used: in particular, the use of a patterned cathode layer that defines the extent of the cell in a particular dimension. We could equally have designed our devices such that each cell was instead defined by the dimensions of a pattern in the ITO anode layer. This would have allowed a continuous cathode layer, which would have eliminated or significantly delayed - the ingress of environmental water at the edges of the cell. Hence, the linear decay with $\sqrt{t}$ as seen in Figure 10(a) and (b) are not universal relationships that hold for all lateral device designs. In general, $I_{s c}$ (and also $1 / R_{\text {se }}$ ) decays due to diffusion from the edges inwards as

$$
I_{s c}=k_{1} t-k_{2} \sqrt{t}+k_{3}
$$

where $k_{1}, k_{2}$ and $k_{3}$ are constants depending on $a$, the dimensions of the active area and lateral device design.

To illustrate the improvement in lifetime that could be achieved, we have calculated the expected time decay of $I_{\mathrm{sc}}, R_{\mathrm{se}}$ and efficiency for our $2 \mathrm{~mm} \times 10 \mathrm{~mm}$ OPV devices, if the cathode layer was extended beyond the cell dimension in various different combinations. While the photoactive dimensions of these devices are the same, the perimeter length that is defined by the cathode is different for each of the 8 lateral device designs. The results are presented in Fig. 11. Here, we have taken a value for the rate prefactor, $a$, from our experiments reported above, and assumed that the devices are not subject to degradation by any other mechanism, including the ingress of water through pinholes. This is, of course, not the case; but it is useful for illustrating the relative effect of the primary mode of degradation. Knowing how $I_{s c}$ and $R_{s e}$ change over time, efficiency values have been 
determined assuming the classical one-diode model for photovoltaics. The shunt resistance is assumed to be constant with time, as suggested by I-V measurements, and the diode ideality factor was also held constant. For a device of our design, the most durable case is one in which the cathode size and shape permits water ingress only at one of the $2 \mathrm{~mm}$ edges of the device, which reduces efficiency by $15 \%$ over the first 5.5 hours. In the worst case, the cell edges are defined entirely by the cathode pattern, thus permitting water ingress at all four edges, resulting in a drop in efficiency of $99.5 \%$ over the same time period. While these explicit decay rates are specific to $2 \mathrm{~mm} \times 10 \mathrm{~mm}$ cells, the concept remains important for all research-scale OPV devices of this axial design.

For the best case described here, i.e. edge-diffusion at one short edge only, the rate of degradation is slow enough that we would expect degradation at pinholes to dominate. This explains why others have reported pinhole-dominated degradation. For example,

Seeland et al. [11], used electroluminescence imaging to demonstrate a degradation pattern consistent with water ingress through pinholes, rather than from edges. In that study, devices of the same axial structure (i.e. ITO/PEDOT:PSS/P3HT:PCBM/aluminium) had a lifetime that was an order of magnitude higher than the results presented here. On the basis of our results, we argue that the choice of a particular lateral device design can strongly influence the rate and the pattern of device degradation and should be discussed whenever results of OPV cell degradation are reported, especially when devices with different lateral device designs are compared.

(a)

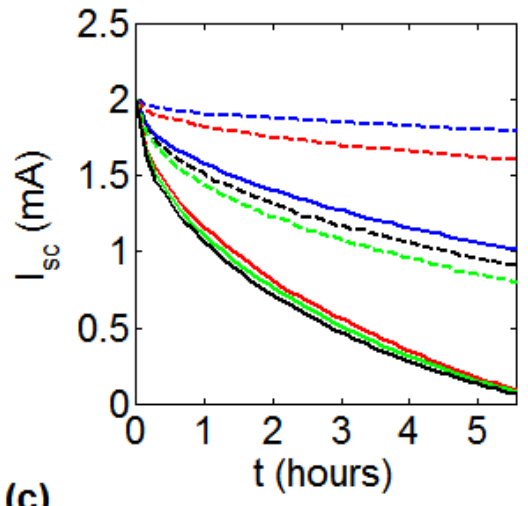

(c)

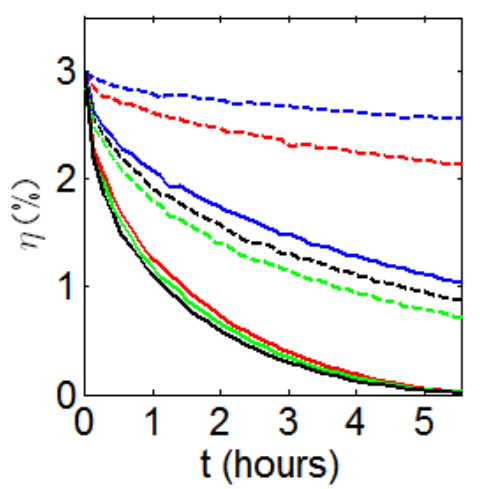

(b)

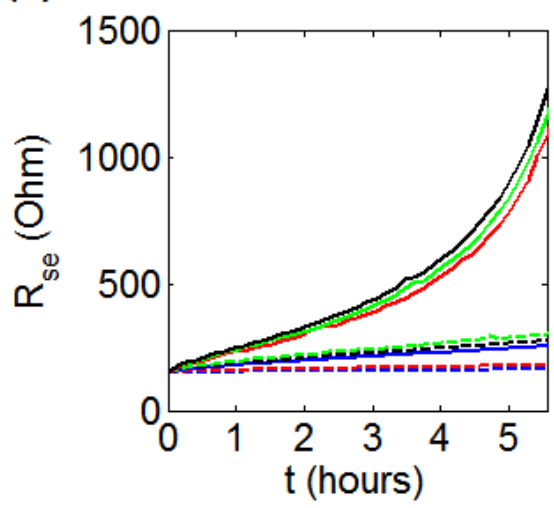

----- perimeter length $2 \mathrm{~mm}$ perimeter length $4 \mathrm{~mm}$

- perimeter length $10 \mathrm{~mm}$

----- perimeter length $12 \mathrm{~mm}$

----- perimeter length $14 \mathrm{~mm}$

- perimeter length $20 \mathrm{~mm}$ perimeter length $22 \mathrm{~mm}$

- perimeter length $24 \mathrm{~mm}$

Figure 11: Predictions for the decay of short-circuit current (a), series resistance (b) and cell efficiency (c) for a $2 \mathrm{~mm} \times 10 \mathrm{~mm}$ OPV cell of the type used for this study. The eight curves correspond to the eight possible anode-cathode configurations for a rectangular device 
without changing the cell dimensions. Specifically, dashed blue represents a cathode allowing water ingress at one short edge only; dashed red, at the two short edges; solid blue, at one long edge; dotted red, at one short edge and one long edge; dotted green, at two short edges and one long edge; solid red, at two long edges; solid green, at two long edges and one short edge; and solid black, at all edges of the device. The corresponding perimeter length of the photoactive area that is defined by the cathode is shown in the legend.

\section{Conclusions}

We have used a photocurrent mapping technique to study degradation features in organic solar cells with the common axial structure of ITO/PEDOT:PSS/(P3HT-PCBM)/cathode, in which the primary degradation mechanism is the oxidation of the aluminium cathode due to water ingress. This oxidation leads to complete local inhibition of charge extraction as well as an increase in the series resistance and hence fill-factor of the device. Over most of its area the cathode layer is protective against water ingress, however water enters the device via diffusion through pinholes in the layer, and also at the edge. This effect is accelerated by the presence of the hygroscopic PEDOT:PSS layer. Using a model for diffusion into the device we have established that the increase of the diffusion rate due to a $35 \mathrm{~nm}$ PEDOT:PSS layer is approximately a factor of 10 .

We see evidence that degradation at pinholes occurs more slowly than at the device edge, and that a larger pinhole leads to a higher rate of local degradation near the pinhole, compared with a smaller pinhole. We find this reasonable on the basis that the diffusive ingress of water through pinholes is likely to be limited to a degree by the conductance of the pinhole aperture. Since pinholes in the cathode exhibit a distribution of sizes, their role in degradation varies, however even for the largest pinhole in our devices, the rate of water ingress is lower than occurs at the cathode edge by a factor of approximately 3 .

We observe that degradation at the edges of a cell may be significantly slowed by designing the device such that the cathode layer extends beyond the edge of the active cell, i.e. by defining the edges of the cell with the ITO pattern, rather than the cathode pattern. This would significantly increase the time taken for water to reach the active area of the cell, and explains why some authors report degradation at pinholes as more important.

Our diffusion calculation was used to determine the diffusivity of water in PEDOT:PSS to be $(5.0 \pm 2.7) \times 10^{-6} \mathrm{~cm}^{2} \mathrm{~s}^{-1}$. The determination of this value did not exclusively require complete local degradation of cell performance; the method also worked for devices with a silver cathode, where we instead tracked the path of a localised region showing improved cell performance. We attribute this small improvement to the formation of a temporary sub-oxide of silver that moves through the device along with the front of water ingress. The applicability of the method to more than one cathode material suggests it may be useful for a range of OPV device types, both traditional and inverted, and may provide a useful tool for studying the performance of encapsulation in devices close to the commercialisation stage. 
Finally, the diffusion calculation was combined with the classical one-diode model for photovoltaics to model the impact of lateral device design on the overall cell performance. We quantify the sensitivity of the device lifetime to the placement of the cathode edge. It is clear that the lateral architecture of the device affects its durability and hence any reported durability results should be placed in the context of a specific device design.

\section{Acknowledgements}

The authors gratefully acknowledge funding for this work through CSIRO Energy Technology and CSIRO's Future Manufacturing Flagship.

\section{References}

[1] M.A. Green, K. Emery, Y. Hishikawa, W. Warta, E.D. Dunlop, Solar cell efficiency tables (version 39), Progress in Photovoltaics: Research and Applications 20 (2012) 12-20.

[2] M. Jørgensen, K. Norrman, S. a Gevorgyan, T. Tromholt, B. Andreasen, F.C. Krebs, Stability of polymer solar cells., Advanced Materials 24 (2012) 580-612.

[3] M.O. Reese, A.J. Morfa, M.S. White, N. Kopidakis, S.E. Shaheen, G. Rumbles, et al., Pathways for the degradation of organic photovoltaic P3HT:PCBM based devices, Solar Energy Materials and Solar Cells 92 (2008) 746-752.

[4] K. Norrman, N.B. Larsen, F.C. Krebs, Lifetimes of organic photovoltaics: Combining chemical and physical characterisation techniques to study degradation mechanisms, Solar Energy Materials and Solar Cells 90 (2006) 2793-2814.

[5] K. Kawano, R. Pacios, D. Poplavskyy, J. Nelson, D.D.C. Bradley, J.R. Durrant, Degradation of organic solar cells due to air exposure, Solar Energy Materials and Solar Cells 90 (2006) 3520-3530.

[6] E. Voroshazi, B. Verreet, A. Buri, R. Müller, D. Di Nuzzo, P. Heremans, Influence of cathode oxidation via the hole extraction layer in polymer:fullerene solar cells, Organic Electronics 12 (2011) 736-744.

[7] M. Hermenau, M. Riede, K. Leo, S. a. Gevorgyan, F.C. Krebs, K. Norrman, Water and oxygen induced degradation of small molecule organic solar cells, Solar Energy Materials and Solar Cells 95 (2011) 1268-1277.

[8] K. Norrman, S. a Gevorgyan, F.C. Krebs, Water-induced degradation of polymer solar cells studied by H2(18)O labeling., ACS Applied Materials and Interfaces 1 (2009) 10212.

[9] M. Jørgensen, K. Norrman, F.C. Krebs, Stability/degradation of polymer solar cells, Solar Energy Materials and Solar Cells 92 (2008) 686-714. 
[10] F.C. Krebs, K. Norrman, Analysis of the failure mechanism for a stable organic photovoltaic during $10000 \mathrm{~h}$ of testing, Progress in Photovoltaics: Research and Applications 15 (2007) 697-712.

[11] M. Seeland, R. Rösch, H. Hoppe, Luminescence imaging of polymer solar cells: Visualization of progressing degradation, Journal of Applied Physics 109 (2011) 064513.

[12] M. Hermenau, S. Schubert, H. Klumbies, J. Fahlteich, L. Müller-Meskamp, K. Leo, et al., The effect of barrier performance on the lifetime of small-molecule organic solar cells, Solar Energy Materials and Solar Cells 97 (2012) 102-108.

[13] T. Jeranko, H. Tributsch, N.S. Sariciftci, J.C. Hummelen, Patterns of efficiency and degradation of composite polymer solar cells, Solar Energy Materials and Solar Cells 83 (2004) 247-262.

[14] R. Rösch, D.M. Tanenbaum, M. Jørgensen, M. Seeland, M. Bärenklau, M. Hermenau, et al., Investigation of the degradation mechanisms of a variety of organic photovoltaic devices by combination of imaging techniques - the ISOS-3 interlaboratory collaboration, Energy and Environmental Science 5 (2012) 6521.

[15] G. Li, C.-W. Chu, V. Shrotriya, J. Huang, Y. Yang, Efficient inverted polymer solar cells, Applied Physics Letters 88 (2006) 253503.

[16] B.J. Leever, M.F. Durstock, M.D. Irwin, a. W. Hains, T.J. Marks, L.S.C. Pingree, et al., Spatially resolved photocurrent mapping of operating organic photovoltaic devices using atomic force photovoltaic microscopy, Applied Physics Letters 92 (2008) 013302.

[17] D.C. Coffey, O.G. Reid, D.B. Rodovsky, G.P. Bartholomew, D.S. Ginger, Mapping local photocurrents in polymer/fullerene solar cells with photoconductive atomic force microscopy., Nano Letters 7 (2007) 738-44.

[18] C.R. McNeill, H. Frohne, J.L. Holdsworth, J.E. Furst, B.V. King, P.C. Dastoor, Direct Photocurrent Mapping of Organic Solar Cells Using a Near-Field Scanning Optical Microscope, Nano Letters 4 (2004) 219-223.

[19] O. Breitenstein, M. Langenkamp, Lock-in Thermography - Basics and Use for Functional Diagnostics of Electronic Components, Springer, Berlin, 2003.

[20] M. Acciarri, S. Binetti, A. Racz, S. Pizzini, G. Agostinelli, Fast LBIC in-line characterization for process quality control in the photovoltaic industry, Solar Energy Materials and Solar Cells 72 (2002) 417-424.

[21] F.C. Krebs, R. Søndergaard, M. Jørgensen, Printed metal back electrodes for R2R fabricated polymer solar cells studied using the LBIC technique, Solar Energy Materials and Solar Cells 95 (2011) 1348-1353. 
[22] Y. Galagan, B. Zimmermann, E.W.C. Coenen, M. Jørgensen, D.M. Tanenbaum, F.C. Krebs, et al., Current Collecting Grids for ITO-Free Solar Cells, Advanced Energy Materials 2 (2012) 103-110.

[23] J.M. Kroon, M.M. Wienk, W.J.H. Verhees, J.C. Hummelen, Accurate efficiency determination and stability studies of conjugated polymer/fullerene solar cells, Thin Solid Films 403-404 (2002) 223-228.

[24] F.C. Krebs, J. Fyenbo, D.M. Tanenbaum, S. a. Gevorgyan, R. Andriessen, B. van Remoortere, et al., The OE-A OPV demonstrator anno domini 2011, Energy and Environmental Science 4 (2011) 4116.

[25] D.M. Tanenbaum, H.F. Dam, R. Rösch, M. Jørgensen, H. Hoppe, F.C. Krebs, Edge sealing for low cost stability enhancement of roll-to-roll processed flexible polymer solar cell modules, Solar Energy Materials and Solar Cells 97 (2012) 157-163.

[26] S. Harris, Microscopic theory for the diffusive evolution of an isoconcentration surface, Physical Review A 42 (1990) 3504-3506.

[27] A.L. Iordanskii, G.E. Zaikov, The General Aspects of Diffusion of Water in Polymers, International Journal of Polymeric Materials 24 (1994) 193-209.

[28] F.A. Long, L.J. Thompson, Diffusion of water vapor in polymers, Journal of Polymer Science 15 (1955) 413-426.

[29] F. Beck, U. Barsch, The role of water in the electrodeposition and doping of polythiophene and two of its derivatives, Macromolecular Chemistry and Physics 194 (1993) 2725-2739.

[30] W. Wagner, A. Pruss, International Equations for the Saturation Properties of Ordinary Water Substance. Revised According to the International Temperature Scale of 1990., Journal of Physical and Chemical Reference Data 22 (1993) 783-787.

[31] C.-F. Lin, S.-W. Liu, W.-F. Hsu, M. Zhang, T.-L. Chiu, Y. Wu, et al., Modification of silver anode and cathode for a top-illuminated organic photovoltaic device, Journal of Physics D: Applied Physics 43 (2010) 395101.

[32] W.-J. Yoon, P.R. Berger, 4.8\% efficient poly(3-hexylthiophene)-fullerene derivative (1:0.8) bulk heterojunction photovoltaic devices with plasma treated $\mathrm{AgO}$ [sub x]/indium tin oxide anode modification, Applied Physics Letters 92 (2008) 013306.

[33] U. Kumar Barik, S. Srinivasan, C.. Nagendra, a Subrahmanyam, Electrical and optical properties of reactive DC magnetron sputtered silver oxide thin films: role of oxygen, Thin Solid Films 429 (2003) 129-134. 